\title{
Advances in clinical trials of targeted therapy and immunotherapy of lung cancer in 2018
}

\author{
Zhengyang Hü, Ming Li", Zhencong Chen, Cheng Zhan, Zongwu Lin, Qun Wang \\ Department of Thoracic Surgery, Zhongshan Hospital, Fudan University, Shanghai 200032, China \\ Contributions: (I) Conception and design: C Zhan, Z Lin, Q Wang; (II) Administrative support: Z Lin, Q Wang; (III) Provision of study materials or \\ patients: None; (IV) Collection and assembly of data: Z Hu, M Li, C Zhan, Z Chen; (V) Data analysis and interpretation: Z Hu, M Li, C Zhan, Z \\ Chen; (VI) Manuscript writing: All authors; (VII) Final approval of manuscript: All authors. \\ \#These authors contributed equally to this work. \\ Correspondence to: Cheng Zhan, MD; Zongwu Lin, MD. Department of Thoracic Surgery, Zhongshan Hospital, Fudan University; 180 Fenglin Road, \\ Shanghai 200032, China. Email: czhan10@fudan.edu.cn; lin.zongwu@zs-hospital.sh.cn.
}

\begin{abstract}
There were many clinical studies on lung cancer in 2018. In particular, significant progress has been made in immunotherapy and targeted therapy. Whether in small cell lung cancer (SCLC) or nonsmall cell lung cancer (NSCLC), immune checkpoint inhibitors (ICIs) have shown good results. For patients with specific gene mutations, the new generation inhibitors also showed good results in clinical trials. In this review, we summarize the clinical trials in lung cancer in 2018 and describe the progress and prospects for lung cancer therapies.
\end{abstract}

Keywords: Non-small cell lung cancer (NSCLC); small cell lung cancer (SCLC); immunotherapy; targeted therapy; clinical trails

Submitted Jul 12, 2019. Accepted for publication Oct 12, 2019.

doi: $10.21037 /$ tlcr.2019.10.17

View this article at: http://dx.doi.org/10.21037/tlcr.2019.10.17

\section{Introduction}

Among the new cases of malignant tumors and tumor deaths worldwide in 2018 , the incidence $(11.6 \%)$ and mortality (18.4\%) of lung cancer ranked first (1). In China, the incidence and mortality of lung cancer has gradually increased over the past 30 years, and this trend is expected to continue (2). The malignant tumor incidence and mortality study in China in 2015 showed that the incidence and mortality of lung cancer were the highest of any cancers (3). Lung cancer has become one of the problems that threatens human health worldwide. But at the same time, there were many clinical trials that made significant progress in the treatment of lung cancer in 2018. Programmed cell death protein-1 (PD-1) inhibitors, programmed cell death ligand protein-1 (PD-L1) inhibitors, and cytotoxic T lymphocyteassociated antigen-4 (CTLA-4) inhibitors showed good results, both in small cell lung cancer (SCLC) and non- small cell lung cancer (NSCLC). For inoperable patients with gene mutations, targeted therapy has shown superior efficacy compared to traditional chemotherapy. Targeted therapy and immunotherapy may therefore become the first-line treatments for inoperable NSCLC and SCLC.

\section{Targeted therapy}

Among all NSCLC patients, approximately $30-40 \%$ of Asian patients and $10-20 \%$ of Caucasian patients have epidermal growth factor receptor (EGFR) gene mutations, while approximately $10 \%$ of Asian patients and $30 \%$ of Caucasian patients have Kirsten rat sarcoma viral oncogene (KRAS) mutations, and $1-7 \%$ and $1.7 \%$ of patients have anaplastic lymphoma kinase (ALK) and ROS1 gene mutations, respectively. A small number of patients also have human epidermal growth factor receptor-2 (HER2) gene mutations (4). 


\section{The EGFR gene mutation}

Compared with chemotherapy, EGFR tyrosine kinase inhibitors (TKIs) not only show better efficacy, but also have the advantages of oral administration, better compliance, and lower toxicity (5). Whether for local advanced or metastatic NSCLC patients, or for postoperative adjuvant therapy, using tissues or peripheral blood for molecular testing to determine EGFR mutations, and then using EGFR-TKIs as recommended by the NCCN guidelines as a first or second-line therapy is suggested (6). Since the first EGFR-TKI, gefitinib, was used at the beginning of this century, new generation EGFR-TKIs, including afatinib and osimertinib have been used successfully. Clinical trials for those drugs occurred in 2018 (Table 1).

\section{First generation EGFR-TKIs}

In a phase IV clinical study (NCT01609543) (7) of erlotinib as the first-line treatment, a total of 62 patients were treated with this drug. The objective response rate (ORR) was $66.1 \%$, and the median progression-free survival (mPFS) was 12.8 months. Although determination of the overall survival (OS) was premature, the 1-year survival was $82.5 \%$, which was a significant improvement compared with traditional chemotherapy having a remission rate of $20-35 \%$ and median survival time of $10-12$ months (20). As for second-line treatment, the ORR of erlotinib was $25.5 \%$, the mPFS was 4.8 months, and the OS was 10.4 months (8). Compared with vinorelbine and cisplatin as the postoperative adjuvant chemotherapy for stage IIIA NSCLC patients, the median disease-free survival was doubled in the erlotinib group (42.2 vs. 21.0 months, $\mathrm{P}=0.0054)$. The 2 - and 3 -year disease-free survival rate also increased significantly at the same time $(81.4 \%$ vs. $44.6 \%$, $\mathrm{P}=0.0054 ; 54.2 \%$ vs. $19.8 \%, \mathrm{P}=0.0460$, respectively) (9).

In another clinical study comparing the effects of EGFR-TKIs and chemotherapy as first-line therapies (NCT00997230) (10), 53\% of all 334 patients chose gefitinib. Gefitinib's mPFS was longer than that of chemotherapy (10.0 vs. 7.0 months, $\mathrm{P}=0.022$ ), and the mOS was also extended to 4.5 months (18.1 vs. 13.6 months, $\mathrm{P}=0.005)$. However, in a study by Yang et al. (11), gefitinib combined with platinum had no statistical difference in the mPFS and ORR compared with using platinum alone. The combination was superior only in the mOS (18.6 vs. 14.9 months). Uchibori et al. (12) studied the efficacy of gefitinib in combination with pemetrexed as the second-line treatment after using gefitinib. The results showed that the mPFS was 6.7 months and the mOS was 24.3 months, which meant a combination of the two drugs could be used for patients who could not be treated with platinum and did not have the T790M mutation after first-line gefitinib treatment.

\section{Second generation EGFR-TKIs}

The second-generation inhibitors represented by afatinib are characterized by irreversible binding to the EGFR mutant. In addition to competitively occupying the ATP binding site on EGFR, they can also alkylate or covalently bond with specific amino acid residues near the EGFR binding site, which can dramatically increase the drug concentration, to provide persistent blocking and enhancement of the tumor cell inhibition (21).

Some studies compared the effects of first-generation inhibitors, such as gefitinib and erlotinib, with the secondgeneration inhibitor, afatinib, as a first-line therapy. In a retrospective study, Fujiwara et al. (13) found no significant difference in the failure time (gefitinib: 9.2 months; erlotinib: 9.8 months; and afatinib: 13.1 months) and mPFS (gefitinib: 27.3 months; erlotinib: 29.3 months; and afatinib not reported) for these three drugs. However, in another study, Tu et al. (14) found that the mPFS of afatinib was longer than that of gefitinib (12.2 vs. 9.8 months, $\mathrm{P}=0.035)$, but similar to erlotinib (12.2 vs. 11.4 months, $\mathrm{P}=0.38$ ). Afatinib had a longer mPFS in a subgroup of patients without brain metastasis (afatinib: 13.1 months; gefitinib: 9.8 months; and erlotinib: 11.7 months; $\mathrm{P}=0.010$ ).

Compared with traditional chemotherapy, the first- and second-generation EGFR-TKIs have significant effects in patients with EGFR gene mutations, thus they are considered as first-line treatment. However, the effects between them still need to be further compared.

\section{Third generation EGFR-TKIs}

A meta-analysis showed that the mPFS using gefitinib or erlotinib as first-line treatments was 11 months (22). The main cause of tumor progression $(\geq 50 \%)$ occurred when the threonine790 of the EGFR gene was replaced by methionine (T790M) (23). The T790M mutation weakened the binding ability of gefitinib or erlotinib to EGFR-TKI and increased the affinity of EGFR for ATP by altering the EGFR spatial conformation (24).

Osimertinib is a selective, irreversible combination third generation inhibitor. It is sensitive not only to EGFR mutations, but also to T790M mutations $(24,25)$. Previous AURA series studies $(26,27)$ and other trials $(28,29)$ showed 


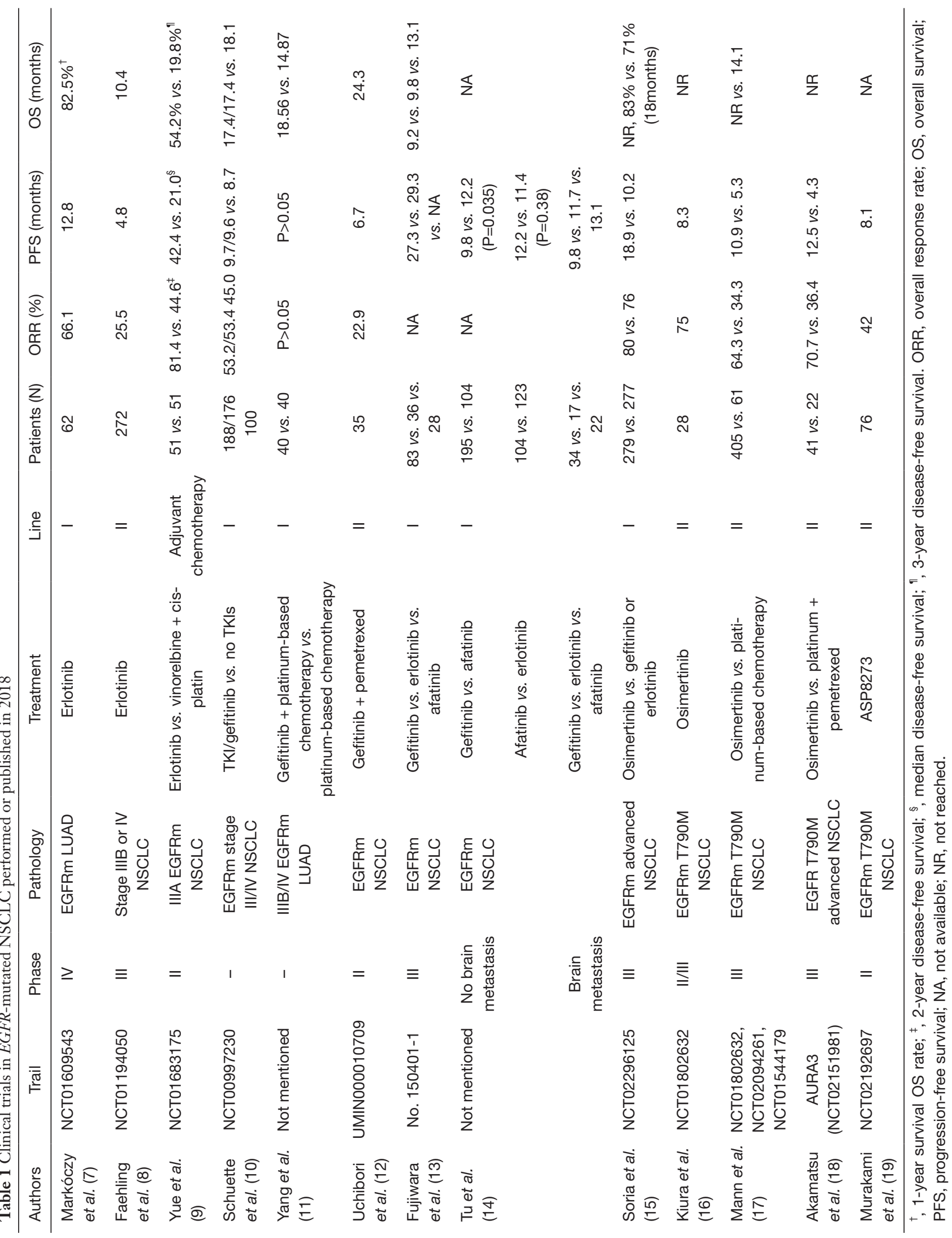


that it was an effective first- or second-line treatment for EGFR mutant NSCLC, even when compared with first generation EGFR-TKIs. However, osimertinib had a better ability to penetrate the blood-brain barrier (30). Thus, osimertinib is the first choice for disease progression with the T790M mutation after treatment with EGFR-TKIs.

In a clinical trial (NCT02296125) (15), 279 patients received osimertinib and 277 received the standard EGFRTKIs (gefitinib or erlotinib). The mPFS in the osimertinib group was prolonged by nearly 8.7 months (18.9 vs. 10.7 months, $\mathrm{P}<0.001)$, and fewer brain metastases were observed ( $6 \%$ vs. 15\%). In terms of disease control rate (DCR), both groups reached $90 \%$ ( $97 \%$ vs. $92 \%$ ) or more and the ORR of osimertinib was slightly higher, but had no statistical significance $(80 \%$ vs. $76 \%, \mathrm{P}=0.24)$. Before the end of the trial, OS was not yet determined, but osimertinib treatment was much safer. Therefore, in patients with EGFR mutations, osimertinib can be considered as a first-line therapy.

In the remaining studies on osimertinib as a secondline treatment, Kiura et al. (16) found that the ORR of osimertinib was $75 \%$, and the mPFS was 8.3 months. Mann et al. (17) compared the effects of osimertinib in the AURA and AURA2 trials and the effects of platinum in the IMPRESS trial. In these trials, patients had the T790M mutation. Osimertinib had a longer PFS (mPFS: 10.9 vs. 5.3 months, $\mathrm{P}<0.0001)$, better ORR (64.3\% vs. $34.3 \%)$, and better DCR (92.1\% vs. $75.0 \%)$. Although the OS of the osimertinib group has not been reached, it was significantly improved compared to platinum ( $\mathrm{HR}=0.412, \mathrm{P}<0.0001)$. Akamatsu et al. (18) studied the efficacy of Japanese patients in the AURA3 trial, where osimertinib also showed better results than platinum (ORR $70.7 \%$ vs. $36.4 \%$; mPFS 12.5 vs. 4.3 months).

Although osimertinib showed good results as a first- or second-line therapy, with the widespread use of osimertinib, the problem of drug resistance has gradually emerged. Studies including FLAURA indicated that the most common resistance mechanisms for osimertinib was MET amplification (15\%) and EGFR C797S mutation (7\%). Others resistance mechanisms included HER2 amplification (2\%), PIK3CA, (7\%) and RAS mutations, while no T790M mutations were found $(31,32)$. The new drug, EAI045, has been successful in mice against osimertinib resistance (33).

Whether as first- or second-line treatment, osimertinib has shown good potential. Thus, using osimertinib as firstline treatment or the sequential therapy after the drug resistant of the first-generation TKIs, is the focus of the further studies.
Regarding another T790M mutation inhibitor, ASP8273, in a phase I and II trial (NCT02192697) (19) involving Asian patients the drug provided an ORR of $42 \%$ and a PFS of 8.1 months. Although ASP8273 showed some effect, there was still a gap compared with osimertinib.

\section{ALK rearrangement}

The $A L K$ gene mutation is caused by inversion of the short arm of the second chromosome, making the EML4 gene and the $A L K$ gene form the EML4-ALK fusion gene, which results in a key tumorigenic driver (34). Compared with chemotherapy, ALK inhibitors, including crizotinib can significantly prolong the survival of patients with $A L K$ gene mutations (35) (Table 2).

\section{First generation ALK rearrangement inhibitors}

Crizotinib, the first ALK-TKI approved by the FDA for the treatment of local advanced or metastatic NSCLC with $A L K$ gene mutations, is effective not only for ALK mutants, but also for ROS1 and cMET kinases. Since 2011, Crizotinib has always been used as the first line of treatment for $A L K$-mutation NSCLC.

In a retrospective cohort study of US patients with $A L K$ mutations, Davies et al. (36) reported that the total OS of patients using crizotinib or ceritinib reached 29.4 months, while it reached 27.1 months in patients with central nervous system (CNS) metastasis, and 36.9 months in patients without CNS metastasis. Nishio et al. (37) reviewed two Phase III clinical trials: PROFILE 1007 (NCT00932893) and PROFILE 1014 (NCT01154140). They found that compared with chemotherapy, if Asian patients were treated with crizotinib as a first-line treatment, the mPFS nearly doubled (13.6 vs. 7.0 months, $\mathrm{P}<0.001$ ) and the ORR was $70 \%$ vs. $54 \%$. In non-Asian patients, the mPFS prolongation was not significant when compared with the Asian group (9.6 vs. 7.2 months, $\mathrm{P}<0.001$ ), with an ORR of $78 \%$ vs. $37 \%$, respectively. As for the second-line treatments, the mPFS was also significantly prolonged in Asian patients (8.1 vs. 2.8 months, $\mathrm{P}<0.001)$; the effect was equally pronounced in non-Asian patients ( 7.1 vs. 3.2 months, $\mathrm{P}<0.001)$. The trial also showed that Crizotinib had a faster onset time, longer duration, and less side effects.

Although the effects of crizotinib are significant, the problem of drug resistance is inevitable. In a retrospective study of 199 patients, the average time to failure with crizotinib as a first-line treatment was 10.4 months and 
Table 2 Clinical trials in ALK-rearranged NSCLC performed or published in 2018

\begin{tabular}{|c|c|c|c|c|c|c|c|c|}
\hline Authors & Trail & Phase & Treatment & Line & Patients (N) & ORR (\%) & PFS (months) & OS (months) \\
\hline \multirow{4}{*}{$\begin{array}{l}\text { Nishio } \\
\text { et al. (37) }\end{array}$} & & & $\begin{array}{l}\text { Previously treated } \\
\text { Asian patients }\end{array}$ & II & $79 / 78$ & $75 / 22$ & $8.1 / 2.8$ & NA \\
\hline & & & $\begin{array}{l}\text { Previously treated } \\
\text { non-Asian patients }\end{array}$ & II & $94 / 96$ & $57 / 18$ & $7.1 / 3.2$ & \\
\hline & & & $\begin{array}{l}\text { Previously untreated } \\
\text { Asian patients }\end{array}$ & I & $77 / 80$ & $70 / 54$ & $13.6 / 7.0$ & \\
\hline & & & $\begin{array}{l}\text { Previously untreated non- } \\
\text { Asian patients }\end{array}$ & -1 & $95 / 91$ & $78 / 37$ & $9.6 / 7.2$ & \\
\hline $\begin{array}{l}\text { Metro } \\
\text { et al. (39) }\end{array}$ & Not mentioned & III & Ceritinib & II & 70 & 40.6 & 8.2 & 15.5 \\
\hline $\begin{array}{l}\text { Hida } \\
\text { et al. (40) }\end{array}$ & $\begin{array}{l}\text { ASCEND-9 } \\
\text { (NCT02450903) }\end{array}$ & II & ceritinib & II & 20 & 25 & 3.7 & $75.6 \%^{\dagger}$ \\
\hline $\begin{array}{l}\text { Kiura } \\
\text { et al. (41) }\end{array}$ & $\begin{array}{l}\text { ASCEND-5 } \\
\text { (NCT01828112) }\end{array}$ & III & $\begin{array}{l}\text { Ceritinib vs. } \\
\text { chemotherapy }\end{array}$ & II & 11 vs. 18 & 54.5 vs. 0 & 9.8 vs. 1.6 & $\begin{array}{l}23.9 \text { vs. } 22.8 \\
(\mathrm{HR}=0.88)\end{array}$ \\
\hline $\begin{array}{l}\text { Gadgeel } \\
\text { et al. (42) }\end{array}$ & NCT02075840 & III & Alectinib vs. crizotinib & $\begin{array}{l}\text { CNS } \\
\text { metastases }\end{array}$ & 64 vs. 58 & 78.6 vs. 40.0 & NR vs. 7.4 & NR \\
\hline $\begin{array}{l}\text { Horn } \\
\text { et al. (44) }\end{array}$ & & & & Prior crizotinib & NA & 69.0 & 9.0 & NA \\
\hline \multirow{3}{*}{$\begin{array}{l}\text { Solomon } \\
\text { et al. (45) }\end{array}$} & NCT01970865 & III & Lorlatinib & 1 & 30 & 90.0 & NA & NA \\
\hline & & & & II or more & 198 & 47.0 & NA & NA \\
\hline & & & Brain metastases & & 81 & 63.0 & NA & NA \\
\hline
\end{tabular}

${ }^{\dagger}, 12$-month OS rate; ${ }^{\ddagger}, 12-$ month PFS rate. NA, not available; NR, not reached.

the OS was 33.8 months (38). The main cause of drug resistance was the production of secondary mutations or amplification of $A L K$ genes (38). Therefore, after the first generation of ALK inhibitors, second generation inhibitors such as alectinib, ceritinib, and rigatinib began to be used.

\section{Second generation ALK rearrangement inhibitors}

Ceritinib, as a new generation ALK rearrangement inhibitor, has shown good results as the first-line treatment compared with chemotherapy in a previous ASCEND-4 study (46) (mPFS: 16.6 vs. 8.1 months; ORR: 74\% vs. $45 \%)$. In a study by Metro et al. (39) who used ceritinib as a second-line treatment for progression after the use of crizotinib, patients had an ORR of $40.6 \%$, mPFS of 8.2 months, and mOS of 15.5 months. In the phase II ASCEND-9 study (NCT02450903) (40), ceritinib was used 
as a second-line treatment for progression after treatment with alectinib. The patient ORR was $20 \%$, mPFS was 3.7 months, and one-year survival was $75.6 \%$. In the phase III ASCEND-5 (NCT01828112) trial (41), 29 Japanese patients had previously undergone crizotinib or platinum treatment, 11 of which subsequently received ceritinib therapy, and 18 received chemotherapy (pemetrexed or docetaxel). Compared with chemotherapy, the ceritinib group had a better ORR ( $54.5 \%$ vs. $0 \%$ ) and mPFS (9.8 vs. 1.6 months), but there was no significant difference in the OS (23.9 vs. 22.8 months, HR =0.88). Although ceritinib has shown good results in first-line treatment, further researches are still needed as second-line therapy.

Alectinib was originally used in advanced NSCLC patients with $A L K$ mutations who had progression after treatment with crizotinib or were resistant to crizotinib. However, in a comparison study in 2017, alectinib showed better results than crizotinib as the first-line treatment (47). In addition, patients with $A L K$ mutations were more likely to have CNS metastases (48). Compared to crizotinib, alectinib has a better ability to cross the blood-brain barrier, and thus, alectinib is more effective in patients with CNS metastases (49). The phase III ALEX trial including 303 patients (42). Investigator-assessed PFS with alectinib was consistent between patients with baseline CNS metastases (HR: $0.40,95 \%$ CI: $0.25-0.64)$ and those without (HR: 0.51, 95\% CI: 0.33-0.80) compared with crizotinib, regardless of prior radiotherapy. The results demonstrated superior CNS activity and significantly delayed CNS progression versus crizotinib in patients with previously untreated, advanced ALK+ NSCLC, irrespective of prior CNS disease or radiotherapy (42).

As a result, alectinib can be used as a first-line treatment to achieve better results, rather than waiting until the disease progresses to the CNS before deciding whether to use it.

Brigatinib is a second-generation inhibitor that was approved in 2017 for treatment of crizotinib resistance or progression after crizotinib treatment. In the phase III ALTA-1L trial (43), the effects of brigatinib and crizotinib were compared as first-line treatments in advanced $A L K$ mutation NSCLC patients. The results showed that the mPFS of the brigatinib group was higher; the 12-month survival rate was $67 \%$ vs. $43 \%(\mathrm{P}<0.001)$, the ORR was $71 \%$ vs. $60 \%$, and the OS was premature. A study also found that brigatinib was not only effective against $A L K$ mutation NSCLCs, but also effective for EGFR mutation NSCLCs, especially for C797S and T790M mutations produced by EGFR-TKIs (50). Therefor brigatinib not only has the significant effects in progression after crizotinib treatment, but also has the potential to become a targeted drug as a fourth generation EGFR-TKI.

In the phase I/II clinical trial of another new ALK inhibitor, ensartinib (X-396), Horn et al. (44) reported that in patients who had not previously received ALK-TKI, the RR was $80 \%$ and the mPFS was 26.2 months. Ensartinib also showed effects in patients who had previously received treatment with crizotinib (RR: 69\%; mPFS: 9.0 months). However, the effects of ensartinib require phase III trials to confirm the results of the initial trial.

\section{Third generation ALK rearrangement inhibitors}

Lorlatinib is a potential third-generation ALK, ROS1 mutation inhibitor that can easily cross the blood-brain barrier. Solomon et al. studied the effect of lorlatinib in a clinical trial (NCT01970865) (45). Of the 30 patients who had not previously received treatment, 27 achieved an objective response, and two of three who had brain metastases had an objective response. The ORR was $47.0 \%$ in 198 patients who had previously received at least one ALK inhibitor, of which 81 patients with brain metastases had an ORR of $63.0 \%$. In each subgroup, 53 patients who had previously received only crizotinib had an ORR of $69.5 \%$, and in 111 of those who had received more than two ALK-TKIs, the ORR was $38.7 \%$. The trial showed that lorlatinib has great potential in I/II/III treatments or in patients with brain metastases. Lorlatinib was not only effective against ALK mutations, but was also useful for ROS1 mutations. In a trial of Asian patients, 12 patients with ALK or ROS1 mutations who had previously progressed after ALK-TKI treatment had an ORR of 64\% and a mPFS of 6.5 months. Of the three patients with intracranial metastases, one patient achieved a complete response and the remaining two had partial responses (51).

Lapatinib has shown good results in patients with brain metastases. However, similar to osimertinib, we still need further researches to prove whether using it as first-line treatment or second-line treatment after disease progression.

\section{Vascular endothelial growth factor receptor (VEGFR) inbibitors}

Apatinib is a VEGFR-2 targeting drug that has had great success in the treatment of advanced gastric cancer. In a phase II trial (NCT02515435) (52,53), Wu et al. determined the efficacy of apatinib in patients with advanced NSCLC 
Table 3 Clinical trials of VEGFR inhibitors in NSCLC performed or published in 2018

\begin{tabular}{lcccccccc}
\hline Authors & Trail & Phase & Treatment & Line & Patients (N) & ORR (\%) & PFS (months) & OS (months) \\
\hline Wu et al. (52) & NCT02515435 & II & Apatinib & II/III & 40 & 13.2 & 3.06 \\
Han et al. (53) & NCT02388919 & III & Anlotinib vs. placebo & III & 296 vs. 143 & 9.2 vs. 0.7 & 5.4 vs. 1.4 & 9.6 vs. 6.3 \\
\hline
\end{tabular}

who had received chemotherapy, but failed or could not tolerate it. The results showed that in 40 patients, the ORR and DCR were $13.2 \%$ and $63.2 \%$, respectively, the mPFS was 3.06 months, and the mOS was 7.69 months. In another phase III clinical study, ALTER 0303 (NCT02388919), 52 of the multi-target-TKI, anlotinib, showed good effects as a third-line treatment of NSCLC. A total of 296 Chinese patients received anlotinib and 143 patients received an equal dose of placebo. Compared with the placebo group, patients treated with anlotinib had a prolonged mOS of more than 3 months (9.6 vs. 6.3 months, $\mathrm{P}=0.002$ ), the mPFS was also extended for 4 months (5.4 vs. 1.4 months, $\mathrm{P}<0.001$ ), and the ORR was increased (9.2\% vs. 0.7\%) (Table 3).

\section{The KRAS gene mutation}

Until 2018, almost all clinical studies using first-generation EGFR-TKIs to treat $K R A S$ mutations ended in failure $(54,55)$. However, in 2018, an in vitro study by Moll et al. (56) reported that the second-generation EGFR-TKI, afatinib inhibited the growth of lung adenocarcinoma tissues that expressed the KRAS gene. This may have been related to the ability of afatinib to more broadly inhibit the activity of the ERBB family than the first generation EGFR-TKIs (57). If afatinib still maintains this effect in subsequent clinical studies, it may be effective in the targeted therapy of KRAS mutations.

\section{Immunotherapy}

\section{PD-1 monoclonal antibodies}

Before the advent of immunosuppressants, the treatment of advanced NSCLC lacking targeted genes such as EGFR and $A L K$ was mainly based on platinum combination chemotherapy (58). With the development of tumor immunology research, ICIs, including PD-1 and PD-L1, have become a popular research topic. In the process of tumor cells escaping from immunity, tumors can inhibit the activation of the PD-1 signal, resulting in reduced T cell activity, so that they can avoid being eliminated by the immune system (59). Investigators therefore developed anti-PD-1 mAb (nivolumab and pembrolizumab) and antiPD-L1 monoclonal antibodies (mAbs) (atezolizumab and durvalumab) to target the immune escape of tumor cells (Table 4).

\section{Nivolumab}

In the Phase III CheckMate 227 (NCT02477826) trial (60), Hellmann et al. studied the relationship between the effects of combining nivolumab (PD-1 $\mathrm{mAb}$ ) with ipilimumab (CTLA-4 mAb) and the PD-L1 expression levels in NSCLC patients with high tumor mutation burdens (TMBs). Compared to the use of Nivolumab alone, the safety and effectiveness of the combination was confirmed in previous phase I trials (68). Patients who had not received chemotherapy were divided into two groups according to their level of PD-L1 expression, and the two groups were randomly assigned 1:1:1 to the three subgroups of nivolumab + ipilimumab, nivolumab, and chemotherapy. The results showed that the mPFS was significantly higher in patients receiving nivolumab + ipilimumab than in the chemotherapy group (7.2 vs. 5.5 months; $\mathrm{P}<0.001)$, and the 1 -year progression-free survival was $42.6 \%$ vs. $13.2 \%$. The ORR of the combination group was $45.3 \%$, while the chemotherapy group was only $26.9 \%$. The validity of these results was confirmed in both subgroups with PD-L1 expression levels above $1 \%$ or below $1 \%$. In patients with a low $\mathrm{TMB}$, the combination group did not prolong the mPFS compared with the chemotherapy group.

Data on using nivolumab alone has not been published. However, the study showed that high TMB patients using nivolumab + ipilimumab as a first-line therapy had better results than chemotherapy, regardless of PD-L1 expression levels, and TMB could also be used as a biomarker to assess possible outcomes. This result was also confirmed in another phase II trial, CheckMate 568, in which nivolumab + ipilimumab were used in combination (69). Another retrospective study of nivolumab confirmed that it was equally useful as a second-line treatment (61). A total of 77 patients in that study had an ORR of $19 \%$, a DCR of $60 \%$, and a mPFS and mOS of 4.0 and 8.0 months, respectively. 
Table 4 Clinical trials of PD-1/PD-L1 in SCLC and NSCLC performed or published in 2018

\begin{tabular}{|c|c|c|c|c|c|c|c|c|}
\hline Authors & Trail & Phase & Treatment & Line & Patients (N) & ORR (\%) & PFS (months) & OS (months) \\
\hline $\begin{array}{l}\text { Hellmann } \\
\text { et al. }(60)\end{array}$ & $\begin{array}{l}\text { CheckMate } 227 \\
\text { (NCT02477826), } \\
\text { NSCLC }\end{array}$ & III & $\begin{array}{l}\text { Nivolumab + ipilimumab vs. } \\
\text { nivolumab + chemotherapy vs. } \\
\text { chemotherapy }^{\ddagger}\end{array}$ & I & $\begin{array}{c}187 \text { vs. } 177 \\
\text { vs. } 186\end{array}$ & NA & $\begin{array}{l}3.2 \text { vs. NA vs. } \\
5.5\end{array}$ & NA \\
\hline $\begin{array}{l}\text { Hellmann } \\
\text { et al. (62) }\end{array}$ & $\begin{array}{l}\text { CheckMate 032, } \\
\text { SCLC }\end{array}$ & III & $\begin{array}{l}\text { Nivolumab + ipilimumab vs. } \\
\text { nivolumab }\end{array}$ & $\begin{array}{l}\text { Low } \\
\text { TMB }\end{array}$ & 27 vs. 45 & $\begin{array}{c}22.2 \% \text { vs. } \\
4.8 \%\end{array}$ & $\begin{array}{l}6.2 \%^{\S} \text { vs. } \\
\quad \text { NR }\end{array}$ & $\begin{array}{l}23.4 \%^{\natural} v s . \\
22.1 \%\end{array}$ \\
\hline $\begin{array}{l}\text { Gandhi } \\
\text { et al. (63) }\end{array}$ & $\begin{array}{l}\text { KEYNOTE-189 } \\
\text { (NCT02578680), } \\
\text { NSCLC }\end{array}$ & III & $\begin{array}{l}\text { Pembrolizumab + chemotherapy vs. } \\
\text { placebo + chemotherapy }\end{array}$ & I & 410 vs. 206 & $\begin{array}{c}47.6 \% \text { vs. } \\
18.9 \%\end{array}$ & 8.8 vs. 4.9 & NR vs. 11.3 \\
\hline $\begin{array}{l}\text { Paz-Ares } \\
\text { et al. (64) }\end{array}$ & $\begin{array}{l}\text { KEYNOTE-407 } \\
\text { (NCT02578680), } \\
\text { SCLC }\end{array}$ & III & $\begin{array}{l}\text { Pembrolizumab + chemotherapy vs. } \\
\text { placebo + chemotherapy }\end{array}$ & I & 278 vs. 281 & $\begin{array}{l}58.4 \% \text { vs. } \\
35.0 \%\end{array}$ & 6.4 vs. 4.8 & 15.9 vs. 11.3 \\
\hline $\begin{array}{l}\text { Socinski } \\
\text { et al. (65) }\end{array}$ & $\begin{array}{l}\text { IMpower150 } \\
\text { (NCT02366143) } \\
\text { NSCLC }\end{array}$ & III & $\begin{array}{l}\text { Atezolizumab + carboplatin + } \\
\text { paclitaxel vs. bevacizumab + } \\
\text { carboplatin + paclitaxel vs. } \\
\text { Atezolizumab + bevacizumab + } \\
\text { carboplatin + paclitaxel }\end{array}$ & I & $\begin{array}{c}348 \text { vs. } 336 \\
\text { vs. } 356\end{array}$ & NA & $\begin{array}{l}\text { NA vs. } 6.8 \\
\text { vs. } 8.3\end{array}$ & $\begin{array}{l}\text { NA vs. } 14.7 \\
\text { vs. } 19.2\end{array}$ \\
\hline
\end{tabular}

${ }^{\dagger}$, PD-L1 expression at least 1\%; ${ }^{\prime}$, PD-L1 expression less than 1\%; ${ }^{\S}, 1$-year PFS rate; ${ }^{\natural}, 1$-year OS rate; ${ }^{+}, 24-m o n t h$ OS rate. NA, not available; NR, not reached; TMB, tumor mutation burden.

For SCLC, the CheckMate 032 trial (62) also demonstrated that nivolumab and ipilimumab were effective in combination and associated with the patient's TMB. In that trial, 156 patients received a combination of nivolumab + ipilimumab and 245 received nivolumab alone. The ORR of the combination group was significantly higher than patients receiving nivolumab alone, and this result was confirmed in three subgroups of TMB that were high (46.2\% vs. 21.3\%), medium (16.0\% vs. $6.8 \%)$, and low (22.2\% vs. $4.8 \%)$. At the same time, whether the high TMB group was treated with monotherapy or combination therapy, the 1-year progression- free survival rate and 1-year survival rate were better than that of the medium or low TMB group. The study also reported that $\mathrm{TMB}$ was not directly related to survival in patients who did not receive immunotherapy.

Combined with the CheckMate 227 and CheckMate 032 trials, we found that the combination of nivolumab and ipilimumab achieved good results in both NSCLC and SCLC patients, and at the same time, the higher the TMB, the better the effects of ICIs. We hope that additional studies will confirm the effects of other ICIs in combination to facilitate immunotherapy as a dual ICI modality. 


\section{Pembrolizumab}

In the previous phase II KEYNOTE-021 trial comparing pembrolizumab with chemotherapy and using chemotherapy alone, it was shown that pembrolizumab had superior efficacy in combination with chemotherapy (70). In the later phase III KEYNOTE-189 trial (63), the effects of pembrolizumab in combination with pemetrexed or platinum and placebo in combination with chemotherapy in patients with advanced, untreated, metastatic, nonsquamous NSCLC were compared. When compared with the placebo, pembrolizumab prolonged the mPFS by nearly 4 months ( 8.8 vs. 4.9 months; $\mathrm{P}<0.001$ ), the 1 -year progression-free survival was $34.1 \%$ vs. $17.3 \%$, the 1 -year overall survival rate was $73.0 \%$ vs. $48.1 \%$, and the ORR was $47.6 \%$ vs. $18.9 \%$. At the main study end point (PFS, OS), pembrolizumab combined with pemetrexed or platinum reduced the risk of death by more than $50 \%$. It was observed to have a better benefit in all subgroups of trials, including those with a PD-L1 expression ratio $<1 \%$.

The KEYNOTE-407 trial (64) focused on the performance of pembrolizumab in combination with chemotherapy in metastatic squamous cell carcinoma. A total of 278 patients received a combination of pembrolizumab, carboplatin and paclitaxel, and 281 patients received an equal amount of placebo, carboplatin and paclitaxel. In a similar manner, the mOS was significantly prolonged in the pembrolizumab group compared with the placebo group (15.9 vs. 11.3 months; $\mathrm{P}<0.001)$. The ORR of the pembrolizumab group was $57.9 \%$, and the chemotherapy group was $38.4 \%$. The mean remission time was $7.7 v$ s. 4.8 months, and the mPFS also increased (6.4 vs. 4.8 months; $\mathrm{P}<0.001$ ). However, there was no significant difference in 1-year survival rate between the high, medium, and low PD-L1 expression ratio subgroups. Although the adverse effect of the pembrolizumab group was slightly higher than that of the chemotherapy group (69.8\% vs. 68.2\%), the overall risk was still greater than the benefit. Overall, the KEYNOTE-189 and KEYNOTE-407 trials showed that the combination of pembrolizumab and chemotherapy can be considered as first-line treatments in patients with advanced metastatic and non-targeted NSCLC without regard to the expression level of PD-L1.

\section{PD-L1 mAbs}

\section{Atezolizumab}

In the IMpower150 trial (65), Socinski et al. studied the effect of atezolizumab in combination with the VEGF antibody bevacizumab for advanced non-squamous NSCLC patients who had not previously received chemotherapy. A total of 356 patients were treated with atezolizumab + bevacizumab + carboplatin + paclitaxel $(\mathrm{ABCP}), 336$ patients received bevacizumab + carboplatin + paclitaxel $(\mathrm{BCP})$, and another 348 patients received atezolizumab + carboplatin + paclitaxel (ACP). The mPFS was 8.3 months in the $\mathrm{ABCP}$ group and 6.8 months in the $\mathrm{BCP}$ group $(\mathrm{P}<0.001)$. Subgroups with low PD-L1 expression levels or no PDL1 expression confirmed the above results. However, in the subgroup with high expression of PD-L1, the efficacy of the $\mathrm{ABCP}$ was more significant (12.6 vs. 6.8 months). In the subgroup with liver metastasis or having KRAS, EGFR, or $A L K$ mutations, the efficacy of $\mathrm{ABCP}$ was also better. Patients receiving $\mathrm{ABCP}$ had a prolongation of the $\mathrm{mOS}$ for nearly 4.5 months (19.2 vs. 14.7 months; $\mathrm{P}=0.02$; the ORR was $63.5 \%$ vs. $48.0 \%)$. The data for the ACP group has not been published.

In the IMpower 133 (NCT02763579) trial (66), Horn et al. studied the effect of atezolizumab in combination with platinum and etoposide as a first-line treatment for metastatic SCLC. A total of 201 patients received atezolizumab and chemotherapy, and 202 patients were treated with placebo and chemotherapy. The mOS of the atezolizumab group was 12.3 months, the placebo group was 10.3 months, and the risk of death was reduced by $30 \%(\mathrm{P}=0.007)$. The 1 -year survival was $51.7 \%$ vs. $38.2 \%$. In a similar manner, the mPFSs were 5.2 and 4.3 months (HR: 0.77 ; 95\% CI: $0.62-0.96$; $\mathrm{P}=0.02$ ) in the atezolizumab and placebo groups, respectively. However, the ORR did not show a significant difference between the two groups $(2.5 \%$ vs. $1.0 \%)$. Analysis of each subgroup found that atezolizumab showed good efficacy, regardless of the TMB level.

Combined with the IMpower150 and IMpower133 trials, we found that the combination of atezolizumab and chemotherapy achieved good results in both NSCLC and SCLC patients. Thus, pembrolizumab and chemotherapy can be considered as first-line treatments in both NSCLC and SCLC patients.

\section{Durvalumab}

In the PACIFIC trial (67) of patients with stage III inoperable NSCLC who had previously received chemoradiotherapy and progressed, 473 patients received durvalumab and 236 patients received a placebo. The 2 -year survival of the durvalumab group was $66.3 \%$, and the placebo group was $55.6 \%$. The mOS was significantly 
Table 5 Clinical trials of monoclonal antibody in lung cancer performed or published in 2018

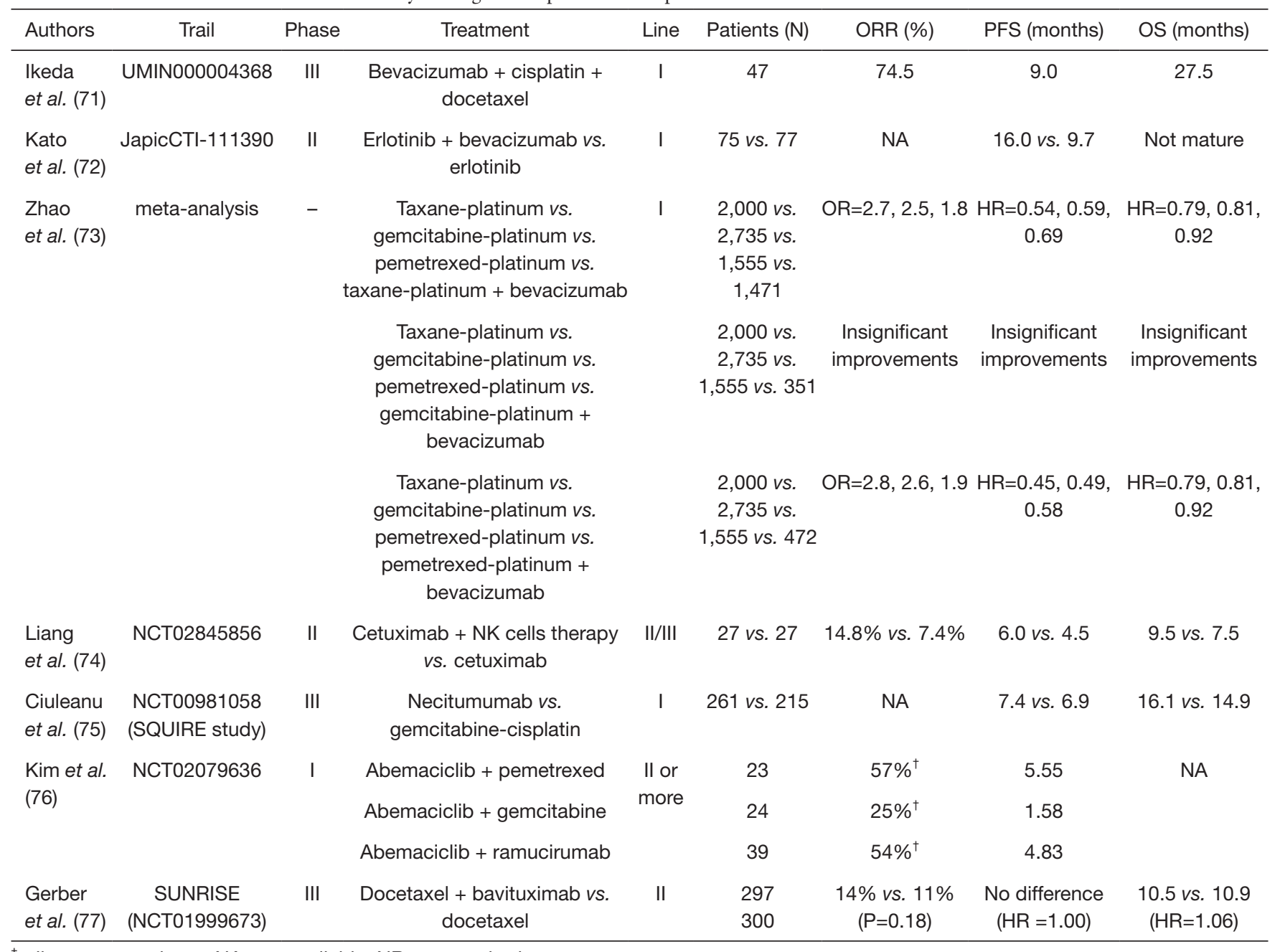

${ }^{\dagger}$, disease control rate. NA, not available; NR, not reached.

prolonged (HR: 0.68, $\mathrm{P}=0.0025)$. These conclusions were observed in each subgroup. In terms of the PFS, durvalumab also showed better results, reaching 17.2 months, compared with 5.6 months in the placebo group (HR: 0.51 ; $95 \%$ CI: $0.41-0.63)$. The ORR of the durvalumab group was $30.0 \%$, and that of the placebo group was $17.8 \%$. The proportion of brain metastases was also lower than that of the placebo group (6.3\% vs. 11.8\%). Although durvalumab showed good results compared to the placebo, the efficacy of durvalumab is yet to be proven compared to chemotherapy or other ICIs.

\section{Other monoclonal antibodies}

In addition to PD-1 mAb and PD-L $1 \mathrm{mAb}$, other monoclonal antibodies like bevacizumab have also shown good results in immunotherapy of lung cancer (Table 5).

\section{Bevacizumab}

Bevacizumab, a monoclonal antibody against VEGFR, has achieved good results in the treatment of various advanced tumors such as colorectal cancer and breast cancer. In the phase II study by Ikeda et al. (71), 41 advanced, non-squamous NSCLC patients received three cycles of bevacizumab in combination with docetaxel and carboplatin, followed by consolidation with bevacizumab. In these patients, the ORR, mPFS, and mOS were $74.5 \%$, 9.0 months, and 27.5 months, respectively. Although bevacizumab showed good results in the trial, $95.7 \%$ of the patients had neutropenia and $59.6 \%$ had leucopenia. 
The high incidence of adverse effects makes it necessary to weigh the pros and cons and early intervention when using this drug in clinical use. Kato et al. (72) studied the efficacy of bevacizumab combined with erlotinib in patients with EGFR mutations (JapicCTI-111390). Compared to erlotinib alone, the combination prolonged the patient PFS (16.0 vs. 9.7 months). A large meta-analysis by Zhao et al. (73) studied the effect of bevacizumab combined with chemotherapy as a first-line therapy. Compared with using paclitaxel-platinum, gemcitabine-platinum, and pemetrexed-platinum, bevacizumab in combination with paclitaxel-platinum or pemetrexed-platinum showed a higher ORR and longer PFS and OS. However, when used in combination with gemcitabine-platinum, it did not show any significant differences.

\section{Cetuximab}

Liang et al. (74) studied the combination of cetuximab and natural killer (NK) cell therapy in the treatment of advanced NSCLC. They found that compared with cetuximab alone, the combination increased the ORR, PFS, and OS (14.8\% vs. $7.4 \%$; 6.0 vs. 4.5 months; 9.5 vs. 7.5 months, respectively), showing that the combination can be an option besides chemoradiotherapy.

\section{Necitumumab}

Necitumumab is an EGFR monoclonal antibody. The effect of necitumumab on stage IV squamous cell carcinoma was reviewed in the SQUIRE study (NCT00981058) (75). Necitumumab in combination with chemotherapy was more effective in patients with EGFR mutations than chemotherapy alone (mOS: 16.1 vs. 14.9 months; HR: 0.76; 95\% CI: 0.61-0.96, $\mathrm{P}<0.05)$. However, the effect on patients without EGFR gene mutation was not significant.

\section{Other immunotherapies}

Abemaciclib, a selective CDK4/6 cell inhibitor, had extensive anti-tumor activity in preclinical trials. It shows better results especially in tumor models with KRAS gene mutations (78). In a trial of stage IV NSCLC (NCT02079636) (76), abemaciclib was used in combination with pemetrexed (AP), gemcitabine (AG), and the VEGFR inhibitor, ramucirumab (AR). The DCR reached $57 \%$ and $54 \%$ in the $\mathrm{AP}$ and $\mathrm{AR}$ groups, respectively, and only $25 \%$ in the AG group. The mPFS of the AP group and the AR group were 5.55 and 4.83 months, respectively, while the AG group was only 1.58 months, which showed that abemaciclib might be better used with pemetrexed or ramucirumab. However, the trial failed to prove the relationship between abemaciclib and KRAS gene mutations.

\section{Immunotherapy failure cases}

In the CheckMate 026 (NCT02041533) (79) study of nivolumab, compared with chemotherapy, using nivolumab as a first-line treatment in advanced NSCLC patients who had PD-L1 expression levels above $5 \%$ did not improve the PFS (4.2 vs. 5.9 months; $\mathrm{P}=0.25$ ), OS (14.4 vs. 13.2 months, HR: 1.02 ; $95 \%$ CI: $0.80-1.30)$ and ORR (26\% vs. $33 \%$ ). Not only nivolumab, but also the PD-L1 monoclonal antibody, duravulumab, were ineffective. In the phase III MYSTIC study, compared with chemotherapy, durvalumab combined with or without CTLA-4 mAb, tremelimumab, did not improve the OS or PFS in patients with stage IV NSCLC. For the phosphatidylserine (PS) $\mathrm{mAb}$, bavituximab, in previous preclinical studies, it was found to inhibit tumor growth, prolong survival, and have a synergistic effect with chemotherapy or radiotherapy (80). The previous phase I and II studies also found that it had a tendency to prolong the survival time of patients $(77,81)$. However, in the SUNRISE (NCT01999673) (82) trial, bavituximab combined with docetaxel had no significant advantage compared with docetaxel alone (ORR: 14\% vs. $11 \% ; \mathrm{P}=0.18$; PFS HR: 1.00; OS HR: 1.06$)$. The potential advantages shown by bavituximab in preclinical trials therefore still needs further research.

The failure of the above clinical trials shows that immunotherapy as a new approach still needs further development, so we must learn from the failures and further explore the best indications for immunotherapy, with a view to giving cancer patients the maximum benefit.

\section{Hyperprogressive disease}

In 2017, Champiat et al. (83) found that in patients who received $\mathrm{PD}-1 / \mathrm{PD}-\mathrm{L} 1$ inhibitors as immunotherapy for 2 months, approximately $9 \%(12 / 131)$ of the patient tumors paradoxically increased by more than $50 \%$ and the rate of progression doubled. This phenomenon is called hyperprogressive disease (HPD). In 2018, Ferrara et al. (84) found in a retrospective study that the probability of HPD in advanced NSCLC patients treated with ICIs was significantly higher than with chemotherapy. Of the 406 patients who received PD-1/PD-L1 inhibitor therapy, 56 (13.8\%) developed HPD and of the 59 patients receiving 
chemotherapy, only three (5.1\%) developed HPD. Among patients receiving ICIs, the mOS in patients with HPD was significantly lower than in patients without HPD (3.4 vs. 6.2 months; HR: 2.18; $\mathrm{P}=0.003)$. The reason for the emergence of HPD is not clear, and may be related to blocking of the PD-1/PD-L1 pathway (85). The high incidence of HPD means that this may be a common pattern of cancer progression, requiring more vigilance in clinical use of ICIs, together with an early assessment of treatment outcomes.

\section{Conclusions}

In 2018, clinical trials of lung cancer yielded impressive results. In terms of targeted therapy, first generation inhibitors are still powerful, but new inhibitors are emerging, challenging the status of such first-generation inhibitors. Brigatinib is effective not only for ALK rearrangement tumor treatment, but also for osimertinib resistance. Lorlatinib is also effective for ALK or ROS1 gene mutations and intracranial transfers.

As for immunotherapy, the CheckMate trial, IMpower trial, and KEYNOTE trial have confirmed that ICIs are effective for both NSCLC and SCLC. In particular, the CheckMate trial indicated that the combination of two ICIs was superior to the use of a single ICI or chemotherapy. The above trials also showed that the effect of immunotherapy had little relationship to the level of PDL1 expression; however, TMB could be used to predict the effect of immunotherapy.

Despite all such positive results, the failure of the CheckMate 026 and MYSTIC trials, and the HDP after immunotherapy has questioned the efficacy of immunotherapy. For patients with mutations, the sequence of targeted therapy and chemotherapy still needs further clinical trials. We expect that with the development of new technologies, more high-level clinical trials will be conducted for lung cancer in the following year, making the treatment of lung cancer more standardized and accurate, and thus, benefiting more patients.

\section{Search strategy and selection criteria}

Data for this Review were identified by searches of PubMed, and references from relevant articles using the search terms "lung cancer", "immune checkpoint inhibitors", "PD-1/ PD-L1 inhibitors", "chemotherapy", "EGFR mutation", "ALK Rearrangement" and other articles. Abstracts and reports from meetings were included only when they related directly to previously published work. Only articles published in English between 2018.1.1 and 2018.12.31 were included.

\section{Acknowledgments}

The authors thank International Science Editing (http:// www.internationalscienceediting.com) for editing this manuscript.

Funding: This work was supported by the National Natural Science Foundation of China (Grant Nos. 81572295; www. nsfc.gov.cn).

\section{Footnote}

Conflicts of Interest: The authors have no conflicts of interest to declare.

Ethical Statement: The authors are accountable for all aspects of the work in ensuring that questions related to the accuracy or integrity of any part of the work are appropriately investigated and resolved.

\section{References}

1. Bray F, Ferlay J, Soerjomataram I, et al. Global cancer statistics 2018: GLOBOCAN estimates of incidence and mortality worldwide for 36 cancers in 185 countries. CA Cancer J Clin 2018;68:394-424.

2. Wang L, Yu C, Liu Y, et al. Lung Cancer Mortality Trends in China from 1988 to 2013: New Challenges and Opportunities for the Government. Int J Environ Res Public Health 2016;13:E1052.

3. Chen $W$, Zheng R, Baade PD, et al. Cancer statistics in China, 2015. CA Cancer J Clin 2016;66:115-32.

4. Li T, Kung HJ, Mack PC, et al. Genotyping and genomic profiling of non-small-cell lung cancer: implications for current and future therapies. J Clin Oncol 2013;31:1039-49.

5. Pirker R, Buder A, Filipits M. Osimertinib in advanced EGFR T790M-positive non-small-cell lung cancer: the clinical impact of AURA3. Transl Cancer Res 2017;6:S265-9.

6. NCCN Clinical Practice Guidelines in Oncology (NCCN Guidelines $\left.{ }^{\circledR}\right)$ Non-Small Cell Lung Cancer (Version 3. 2018).

7. Markóczy Z, Sárosi V, Kudaba I, et al. Erlotinib as single agent first line treatment in locally advanced or metastatic 
activating EGFR mutation-positive lung adenocarcinoma (CEETAC): an open-label, non-randomized, multicenter, phase IV clinical trial. BMC Cancer 2018;18:598.

8. Faehling M, Achenbach J, Staib P, et al. Erlotinib in routine clinical practice for first-line maintenance therapy in patients with advanced non-small cell lung cancer (NSCLC). J Cancer Res Clin Oncol 2018;144:1375-83.

9. Yue D, Xu S, Wang Q, et al. Erlotinib versus vinorelbine plus cisplatin as adjuvant therapy in Chinese patients with stage IIIA EGFR mutation-positive non-small-cell lung cancer (EVAN): a randomised, open-label, phase 2 trial. Lancet Respir Med 2018;6:863-73.

10. Schuette W, Schirmacher P, Eberhardt WEE, et al. Treatment decisions, clinical outcomes, and pharmacoeconomics in the treatment of patients with EGFR mutated stage III/IV NSCLC in Germany: an observational study. BMC Cancer 2018;18:135.

11. Yang L, Fan JH, Liu LL, et al. Comparison of gefitinib and platinum-based chemotherapy and only platinumbased chemotherapy to treat lung adenocarcinoma. J Biol Regul Homeost Agents 2018;32:613-8.

12. Uchibori K, Satouchi M, Sueoka-Aragane N, et al. Phase II trial of gefitinib plus pemetrexed after relapse using first-line gefitinib in patients with non-small cell lung cancer harboring EGFR gene mutations. Lung Cancer 2018;124:65-70.

13. Fujiwara A, Yoshida M, Fujimoto H, et al. A Retrospective Comparison of the Clinical Efficacy of Gefitinib, Erlotinib, and Afatinib in Japanese Patients With Non-Small Cell Lung Cancer. Oncol Res 2018;26:1031-6.

14. Tu CY, Chen C, Liao W, et al. Comparison of the effects of the three major tyrosine kinase inhibitors as firstline therapy for non-small-cell lung cancer harboring epidermal growth factor receptor mutations. Oncotarget 2018;9:24237.

15. Soria JC, Ohe Y, Vansteenkiste J, et al. Osimertinib in Untreated EGFR-Mutated Advanced Non-Small-Cell Lung Cancer. N Engl J Med 2018;378:113-25.

16. Kiura K, Yoh K, Katakami N, et al. Osimertinib in patients with epidermal growth factor receptor T790M advanced non-small cell lung cancer selected using cytology samples. Cancer Sci 2018;109:1177-84.

17. Mann H, Andersohn F, Bodnar C, et al. Adjusted Indirect Comparison Using Propensity Score Matching of Osimertinib to Platinum-Based Doublet Chemotherapy in Patients with EGFRm T790M NSCLC Who Have Progressed after EGFR-TKI. Clin Drug Investig 2018;38:319-31.
18. Akamatsu H, Katakami N, Okamoto I, et al. Osimertinib in Japanese patients withEGFR T790M mutation-positive advanced non-small-cell lung cancer: AURA3 trial. Cancer Sci 2018;109:1930-8.

19. Murakami H, Nokihara H, Hayashi H, et al. Clinical activity of ASP8273 in Asian patients with non-small-cell lung cancer with EGFR activating and T790M mutations. Cancer Sci 2018;109:2852-62.

20. Azim HA, Elattar I, Loberiza FJ, et al. Third generation triplet cytotoxic chemotherapy in advanced non-small cell lung cancer: a systematic overview. Lung Cancer 2009;64:194-8.

21. Li D, Ambrogio L, Shimamura T, et al. BIBW2992, an irreversible EGFR/HER2 inhibitor highly effective in preclinical lung cancer models. Oncogene 2008;27:4702-11.

22. Lee CK, Davies L, Wu YL, et al. Gefitinib or Erlotinib vs Chemotherapy for EGFR Mutation-Positive Lung Cancer: Individual Patient Data Meta-Analysis of Overall Survival. J Natl Cancer Inst 2017;109:djw279.

23. Yu HA, Arcila ME, Rekhtman N, et al. Analysis of tumor specimens at the time of acquired resistance to EGFR-TKI therapy in 155 patients with EGFR-mutant lung cancers. CLIN CANCER RES 2013;19:2240-7.

24. Oxnard GR, Arcila ME, Sima CS, et al. Acquired resistance to EGFR tyrosine kinase inhibitors in EGFRmutant lung cancer: distinct natural history of patients with tumors harboring the T790M mutation. Clin Cancer Res 2011;17:1616-22.

25. Cross DA, Ashton SE, Ghiorghiu S, et al. AZD9291, an irreversible EGFR TKI, overcomes T790M-mediated resistance to EGFR inhibitors in lung cancer. Cancer Discov 2014;4:1046-61.

26. Jänne PA, Yang JC, Kim DW, et al. AZD9291 in EGFR inhibitor-resistant non-small-cell lung cancer. N Engl J Med 2015;372:1689-99.

27. Goss G, Tsai CM, Shepherd FA, et al. Osimertinib for pretreated EGFR Thr790Met-positive advanced nonsmall-cell lung cancer (AURA2): a multicentre, open-label, single-arm, phase 2 study. Lancet Oncol 2016;17:1643-52.

28. Mok TS, Wu YL, Papadimitrakopoulou VA. Osimertinib in EGFR T790M-Positive Lung Cancer. N Engl J Med 2017;376:1993-4.

29. Ramalingam SS, Yang JC, Lee CK, et al. Osimertinib As First-Line Treatment of EGFR Mutation-Positive Advanced Non-Small-Cell Lung Cancer. J Clin Oncol 2018;36:841-9.

30. Goss G, Tsai CM, Shepherd FA, et al. CNS response to osimertinib in patients with T790M-positive advanced 
NSCLC: pooled data from two phase II trials. Ann Oncol 2018;29:687-93.

31. Thress KS, Paweletz C P, Felip E, et al. Acquired EGFR C797S mutation mediates resistance to AZD9291 in nonsmall cell lung cancer harboring EGFR T790M. Nat Med 2015;21:560-2.

32. Chen K, Zhou F, Shen W, et al. Novel Mutations on EGFR Leu792 Potentially Correlate to Acquired Resistance to Osimertinib in Advanced NSCLC. J Thorac Oncol 2017;12:e65-8.

33. Jia Y, Yun CH, Park E, et al. Overcoming EGFR(T790M) and EGFR(C797S) resistance with mutant-selective allosteric inhibitors. Nature 2016;534:129-32.

34. Shaw AT, Yeap BY, Mino-Kenudson M, et al. Clinical features and outcome of patients with non-small-cell lung cancer who harbor EML4-ALK. J Clin Oncol 2009;27:4247-53.

35. Solomon BJ, Mok T, Kim DW, et al. First-line crizotinib versus chemotherapy in ALK-positive lung cancer. N Engl J Med 2014;371:2167-77.

36. Davies J, Martinec M, Coudert M, et al. Real-world anaplastic lymphoma kinase (ALK) rearrangement testing patterns, treatment sequences, and survival of ALK inhibitor-treated patients. Curr Med Res Opin 2019;35:535-42.

37. Nishio M, Kim DW, Wu YL, et al. Crizotinib versus Chemotherapy in Asian Patients with ALK-Positive Advanced Non-small Cell Lung Cancer. Cancer Res Treat 2018;50:691-700.

38. Reynolds C, Masters ET, Black-Shinn J, et al. RealWorld Use and Outcomes of ALK-Positive CrizotinibTreated Metastatic NSCLC in US Community Oncology Practices: A Retrospective Observational Study. J Clin Med 2018;7:E129.

39. Metro G, Passaro A, Lo RG, et al. Ceritinib compassionate use for patients with crizotinib-refractory, anaplastic lymphoma kinase-positive advanced non-small-cell lung cancer. Future Oncol 2018;14:353-61.

40. Hida T, Seto T, Horinouchi H, et al. Phase II study of ceritinib in alectinib-pretreated patients with anaplastic lymphoma kinase-rearranged metastatic non-smallcell lung cancer in Japan: ASCEND-9. Cancer Sci 2018;109:2863-72.

41. Kiura K, Imamura F, Kagamu H, et al. Phase 3 study of ceritinib vs chemotherapy in ALK-rearranged NSCLC patients previously treated with chemotherapy and crizotinib (ASCEND-5): Japanese subset. Jpn J Clin Oncol 2018;48:367-75.
42. Gadgeel S, Peters S, Mok T, et al. Alectinib versus crizotinib in treatment-naive anaplastic lymphoma kinase-positive $(\mathrm{ALK}+)$ non-small-cell lung cancer: CNS efficacy results from the ALEX study. Ann Oncol 2018;29:2214-22.

43. Camidge DR, Kim HR, Ahn MJ, et al. Brigatinib versus Crizotinib in ALK-Positive Non-Small-Cell Lung Cancer. N Engl J Med 2018;379:2027-39.

44. Horn L, Infante JR, Reckamp KL, et al. Ensartinib (X-396) in ALK-Positive Non-Small Cell Lung Cancer: Results from a First-in-Human Phase I/II, Multicenter Study. Clin Cancer Res 2018;24:2771-9.

45. Solomon BJ, Besse B, Bauer TM, et al. Lorlatinib in patients with ALK-positive non-small-cell lung cancer: results from a global phase 2 study. Lancet Oncol 2018;19:1654-67.

46. Soria JC, Tan D, Chiari R, et al. First-line ceritinib versus platinum-based chemotherapy in advanced ALKrearranged non-small-cell lung cancer (ASCEND-4): a randomised, open-label, phase 3 study. Lancet 2017;389:917-29.

47. Peters S, Camidge D R, Shaw A T, et al. Alectinib versus Crizotinib in Untreated ALK-Positive Non-Small-Cell Lung Cancer. N Engl J Med 2017;377:829-38.

48. Johung KL, Yeh N, Desai NB, et al. Extended Survival and Prognostic Factors for Patients With ALK-Rearranged Non-Small-Cell Lung Cancer and Brain Metastasis. J Clin Oncol 2016;34:123-9.

49. Gadgeel SM, Shaw AT, Govindan R, et al. Pooled Analysis of CNS Response to Alectinib in Two Studies of Pretreated Patients With ALK-Positive Non-Small-Cell Lung Cancer. J Clin Oncol 2016;34:4079-85.

50. Uchibori K, Inase N, Araki M, et al. Brigatinib combined with anti-EGFR antibody overcomes osimertinib resistance in EGFR-mutated non-small-cell lung cancer. Nat Commun 2017;8:14768.

51. Lee J, Sun J M, Lee SH, et al. Efficacy and Safety of Lorlatinib in Korean Non-Small-Cell Lung Cancer Patients With ALK or ROS1 Rearrangement Whose Disease Failed to Respond to a Previous Tyrosine Kinase Inhibitor. Clin Lung Cancer 2019;20:215-21.

52. Wu F, Zhang S, Xiong A, et al. A Phase II Clinical Trial of Apatinib in Pretreated Advanced Non-squamous Non-smallcell Lung Cancer. Clin Lung Cancer 2018;19:e831-42.

53. Han B, Li K, Wang Q, et al. Effect of Anlotinib as a Third-Line or Further Treatment on Overall Survival of Patients With Advanced Non-Small Cell Lung Cancer: The ALTER 0303 Phase 3 Randomized Clinical Trial. JAMA Oncol 2018;4:1569-75. 
54. Mao C, Qiu LX, Liao RY, et al. KRAS mutations and resistance to EGFR-TKIs treatment in patients with nonsmall cell lung cancer: a meta-analysis of 22 studies. Lung Cancer 2010;69:272-8.

55. Papadimitrakopoulou V, Lee JJ, Wistuba II, et al. The BATTLE-2 Study: A Biomarker-Integrated Targeted Therapy Study in Previously Treated Patients With Advanced Non-Small-Cell Lung Cancer. J Clin Oncol 2016;34:3638-47.

56. Moll HP, Pranz K, Musteanu M, et al. Afatinib restrains K-RAS-driven lung tumorigenesis. Sci Transl Med 2018; 10:eaao2301.

57. Patricelli MP, Janes MR, Li LS, et al. Selective Inhibition of Oncogenic KRAS Output with Small Molecules Targeting the Inactive State. Cancer Discov 2016;6:316-29.

58. Davies J, Patel M, Gridelli C, et al. Real-world treatment patterns for patients receiving second-line and thirdline treatment for advanced non-small cell lung cancer: A systematic review of recently published studies. PLoS One 2017;12:e0175679.

59. Chen DS, Irving BA, Hodi FS. Molecular pathways: nextgeneration immunotherapy--inhibiting programmed death-ligand 1 and programmed death-1. Clin Cancer Res 2012;18:6580-7.

60. Hellmann MD, Ciuleanu TE, Pluzanski A, et al. Nivolumab plus Ipilimumab in Lung Cancer with a High Tumor Mutational Burden. N Engl J Med 2018;378:2093-104.

61. Shamai S, Merimsky O. Efficacy and safety of Nivolumab in non-small cell lung cancer patients in Tel-Aviv tertiary medical center: Facing the reality. Mol Clin Oncol 2018;9:419-22.

62. Hellmann MD, Callahan MK, Awad MM, et al. Tumor Mutational Burden and Efficacy of Nivolumab Monotherapy and in Combination with Ipilimumab in Small-Cell Lung Cancer. Cancer Cell 2018;33:853-861.e4.

63. Gandhi L, Rodriguez-Abreu D, Gadgeel S, et al. Pembrolizumab plus Chemotherapy in Metastatic NonSmall-Cell Lung Cancer. N Engl J Med 2018;378:2078-92.

64. Paz-Ares L, Luft A, Vicente D, et al. Pembrolizumab plus Chemotherapy for Squamous Non-Small-Cell Lung Cancer. N Engl J Med, 2018;379:2040-51.

65. Socinski MA, Jotte RM, Cappuzzo F, et al. Atezolizumab for First-Line Treatment of Metastatic Nonsquamous NSCLC. N Engl J Med 2018;378:2288-301.

66. Horn L, Mansfield A S, Szczesna A, et al. First-Line Atezolizumab plus Chemotherapy in Extensive-Stage Small-Cell Lung Cancer. N Engl J Med 2018;379:2220-9. 67. Antonia SJ, Villegas A, Daniel D, et al. Overall Survival with Durvalumab versus Placebo after Chemoradiotherapy in Stage III NSCLC. N Engl J Med 2018;379:2342-50.

68. Hellmann MD, Rizvi NA, Goldman JW, et al. Nivolumab plus ipilimumab as first-line treatment for advanced nonsmall-cell lung cancer (CheckMate 012): results of an open-label, phase 1, multicohort study. Lancet Oncol 2017;18:31-41.

69. Ramalingam SS, Hellmann MD, Awad MM, et al. Tumor mutation burden (TMB) as a biomarker for clinical benefit from dual immune checkpoint blockade with nivolumab (nivo) + ipilimumab (ipi) in first-line (1L) non-small cell lung cancer (NSCLC): identification of TMB cutoff from CheckMate 568. Presented at the American Association for Cancer Research 2018 Annual Meeting, Chicago, April 16, 2018.

70. Langer CJ, Gadgeel SM, Borghaei H, et al. Carboplatin and pemetrexed with or without pembrolizumab for advanced, non-squamous non-small-cell lung cancer: a randomised, phase 2 cohort of the open-label KEYNOTE-021 study. Lancet Oncol 2016;17:1497-508.

71. Ikeda S, Kato T, Ogura T, et al. Phase II study of bevacizumab, cisplatin, and docetaxel plus maintenance bevacizumab as first-line treatment for patients with advanced non-squamous non-small-cell lung cancer combined with exploratory analysis of circulating endothelial cells: Thoracic Oncology Research Group (TORG)1016. BMC Cancer 2018;18:241.

72. Kato T, Seto T, Nishio M, et al. Erlotinib Plus Bevacizumab Phase 11 Study in Patients with Advanced Non-small-Cell Lung Cancer (JO25567): Updated Safety Results. Drug Saf 2018;41:229-37.

73. Zhao S, Gao F, Zhang Y, et al. Bevacizumab in combination with different platinum-based doublets in the first-line treatment for advanced nonsquamous non-smallcell lung cancer: A network meta-analysis. Int J Cancer 2018;142:1676-88.

74. Liang S, Lin M, Niu L, et al. Cetuximab combined with natural killer cells therapy: an alternative to chemoradiotherapy for patients with advanced non-small cell lung cancer (NSCLC). Am J Cancer Res 2018;8:879-91

75. Ciuleanu T, Socinski M A, Obasaju C, et al. Efficacy and Safety of Necitumumab Continuation Therapy in the Phase III SQUIRE Study of Patients With Stage IV Squamous Non-Small-Cell Lung Cancer. Clin Lung Cancer 2018;19:130-8.e2.

76. Kim ES, Kelly K, Paz-Ares LG, et al. Abemaciclib in Combination with Single-Agent Options in Patients with Stage IV Non-Small Cell Lung Cancer: A Phase Ib Study. 
Clin Cancer Res 2018;24:5543-51.

77. Gerber DE, Spigel DR, Giorgadze D, et al. Docetaxel Combined With Bavituximab in Previously Treated, Advanced Nonsquamous Non-Small-Cell Lung Cancer. Clin Lung Cancer 2016;17:169-76.

78. Patnaik A, Rosen LS, Tolaney SM, et al. Efficacy and Safety of Abemaciclib, an Inhibitor of CDK4 and CDK6, for Patients with Breast Cancer, Non-Small Cell Lung Cancer, and Other Solid Tumors. Cancer Discov 2016;6:740-53.

79. Carbone DP, Reck M, Paz-Ares L, et al. First-Line Nivolumab in Stage IV or Recurrent Non-Small-Cell Lung Cancer. N Engl J Med 2017;376:2415-26.

80. He J, Luster T A, Thorpe P E. Radiation-enhanced vascular targeting of human lung cancers in mice with a monoclonal antibody that binds anionic phospholipids. Clin Cancer Res 2007;13:5211-8.

81. Gerber DE, Stopeck AT, Wong L, et al. Phase I safety and pharmacokinetic study of bavituximab, a chimeric

Cite this article as: $\mathrm{Hu} Z$, Li M, Chen Z, Zhan C, Lin Z, Wang Q. Advances in clinical trials of targeted therapy and immunotherapy of lung cancer in 2018. Transl Lung Cancer Res 2019;8(6):1091-1106. doi: 10.21037/tlcr.2019.10.17 phosphatidylserine-targeting monoclonal antibody, in patients with advanced solid tumors. Clin Cancer Res 2011;17:6888-96.

82. Gerber D E, Horn L, Boyer M, et al. Randomized phase III study of docetaxel plus bavituximab in previously treated advanced non-squamous non-small-cell lung cancer. Ann Oncol 2018;29:1548-53.

83. Champiat S, Dercle L, Ammari S, et al. Hyperprogressive Disease Is a New Pattern of Progression in Cancer Patients Treated by Anti-PD-1/PD-L1. Clin Cancer Res 2017;23:1920-8.

84. Ferrara R, Mezquita L, Texier M, et al. Hyperprogressive Disease in Patients With Advanced Non-Small Cell Lung Cancer Treated With PD-1/PD-L1 Inhibitors or With Single-Agent Chemotherapy. JAMA Oncol 2018;4:1543.

85. Champiat S, Ferrara R, Massard C, et al. Hyperprogressive disease: recognizing a novel pattern to improve patient management. Nat Rev Clin Oncol 2018;15:748-62. 